\title{
Changing Paradigms in Arthroplasty Practice
}

\author{
Shubhranshu S. Mohanty ${ }^{1,2} \cdot$ Murali Poduval $^{3}$
}

๑ Indian Orthopaedics Association 2021

"Science is a beautiful gift to humanity; we should not distort it."

\section{APJ ABDUL KALAM}

During the last 20-30 years, there has been a tremendous increase in Arthroplasty practice by Indian Orthopods as evidenced by the registry data [1]. Relieving pain, correcting deformity, achieving stability, and gaining mobility remain the hallmark outcomes of these procedures. However, patient satisfaction has not improved drastically and there is a mismatch between outcomes as assessed by the surgeon and those that align with patient expectations.

There have been significant advances in technology to improve the alignment and stability in knee replacement and anatomical and functional positioning of components in the hip, implants have become significantly more sophisticated, durable and implantation techniques more precise. But, infection still remains a concern and newer strategies are needed to keep tackling this devastating outcome of a joint replacement.

We are operating more younger patients than ever before. The demands and expectations of this group of patients are higher and we are reasonably confident that we can meet them with improvements in biomaterials and implants. There is an increase in the use of the cementless knee with younger patients demanding replacement. The long-term survival of the prosthesis is a matter of concern in this younger population and the greater lifetime risk of revision is yet to be studied in detail. Unicondylar knees have also grown in

Shubhranshu S. Mohanty

drssmohantyortho@gmail.com

Murali Poduval

muralipoduval@gmail.com; murali.poduval@tcs.com

1 Present Address: Orthopaedics, King Edward Memorial Hospital, Parel, Mumbai 400012, India

2 Indian Arthroplasty Association, Mumbai, India

3 Present Address: Engineering and Industrial Services (Medical Devices and Diagnostics), Tata Consultancy Services, Mumbai, India popularity as more surgeons are mastering the technique and assuring good results in well selected patients. The exacting technique of aligning a unicondylar knee has led to adoption of robotics first in the unicondylar than in the total knee. There is increasing awareness of knee alignment philosophy and numerous different approaches like kinematic alignment, restricted kinematic alignment, adjusted mechanical alignment etc. are being explored to bring a natural feel to the knee [2].

Indications are often extended to treat the radiology rather than the patient himself. There is often this mismatch which leads to suboptimal outcomes. High flex implants were introduced but it was realized that surgical technique is of paramount importance than the implant itself to improve post-operative range of movements. Navigation helped to improve alignment and ligament balancing but there was no conclusive evidence of its superiority with respect to patient related outcome or decreased revision rates in long term [3]. It is, however, well established that in extra-articular deformities and compromised medullary canals navigation is a very useful tool. Robotics have entered the scene since some years now and are gaining popularity despite their prohibitive costs. At present, it is too early to comment on its superiority over navigation as it is confined to only showing a better alignment and minimizing iatrogenic soft tissue injuries, but cost-effectiveness is not well established [4]. Still the role of Robotics is not well defined in severe deformities seen in our patients requiring bone grafts, augments, sleeves/ cones, or stem extenders.

After the catastrophic failure of large ball metal on metal hips, ceramic bearings gained in popularity. Monoblock ceramic cups required more meticulous surgical technique and were beyond the domain of an average arthroplasty surgeon and hence gradually withdrawn from market. Since head size more than $36 \mathrm{~mm}$ was not found to be of any greater advantage, most surgeons gravitated back with ceramic on highly crosslinked polyethylene. Although the "Forgotten Joint" status is achieved better with hard-on-hard bearing but allowing the ground level activities is more of an approach towards anatomical placement of the acetabular component without any impingement [5]. There is increasing 
understanding of the role of spino-pelvic parameters in fine tuning acetabular component placement in hip arthroplasty [6]. The personalization of hip arthroplasty is thus closer to reality than ever before. Three-dimensional planning is being introduced in both hip and knee arthroplasty allowing surgeons to have greater control over their surgical procedures thus enabling predictable component alignment. The importance of pre surgical planning is greater in these times than ever before, with most of the surgical preparation already done before the patient is on the table. Unpredictability is therefore reduced, and consistency is ensured in surgical technique and outcomes. Minimally invasive approaches like direct anterior approach (DAA) are slowly picking up adding early recovery due to less tissue trauma. But without specialized equipment and tables it is difficult to execute and has potential for catastrophic consequences. Dual mobility is a very promising implant in complex primary and potentially unstable hips, and has been gaining in popularity in recent times.

Over the years, arthroplasty has gradually progressed to a short stay procedure with the understanding that preoptimization and pre-habilitation improve outcomes, minimize hospital stay and consequently reduce peri-operative and post-operative morbidity and mortality. Improvements in pain management and surgical techniques, as well as well tuned protocols are enabling arthroplasty to become a day care, ambulatory procedure. Both hip and knee arthroplasty are no longer in patient only procedures in the USA. India is also progressing towards shorter stay and ambulatory surgery albeit at a slower pace. This has more to so with social dynamics and economics than the ability of the system to provide the same services as that in North America.

Besides prosthetic wear and loosening, the two most important problems faced by arthroplasty surgeons globally as well in this country are periprosthetic fractures and infection. The increased incidence of periprosthetic fractures is a matter of concern due to osteoporosis and high activity. This is likely to increase in future. At least the surgeon needs to be vigilant in preventing it by meticulous surgical technique in overseeing the occurrence of the incidence during surgery. Newer technology to enable early detection and thus prevent catastrophic consequences is the need of the day. The modern generation of implants with modularity and augmentation capabilities come with increased ability to handle these situations, but at a cost that is often prohibitive.

The most dreaded complication of infection has been a nightmare for arthroplasty surgeons. The extended culture of tissue samples has increased the isolation of the causative organism. Though leukocyte esterase, alpha-defensin and next generation sequencing will be the future in diagnosing infection, still it is beyond the reach of an average arthroplasty surgeon and has its own fallacies [7]. Principles of debridement, antibiotics and implant retention have been delineated in literature and works out in early cases when done meticulously following the principles of aggressive debridement. One stage revision is gaining popularity in European countries. There is need of 'Dedicated arthroplasty Infection Care Centers' with a team of Infectious disease specialists, microbiologists with dedicated arthroplasty surgeons and a good laboratory back up. But managing the emerging trend of drug-resistant organisms and looking out for a higher class of antibiotics with least toxicity to the host is still a matter of concern.

The march of the robots in total joint arthroplasty can hardly be ignored. There is no doubt that these robotic assisted surgeries increase precision and component alignment without the surgeon losing control over the surgical procedure. The consistency of the surgical procedure is considerably improved and the need for large instrument trays eliminated. Robots are, however, expensive and have a large footprint in the operating room. It remains to be seen what the eventual benefits in terms of patient outcomes in the mid-term and the long term are. It is inevitable, however, that in the not so far future, robotics and AI assisted devices will be part of our armamentarium. We need to change with the times and use these technologies to the best of our ability to enhance our technique and outcomes. And most important, we must not go away from the basics and fundamental principles of arthroplasty as taught by the masters not only in our practice but also in the teachings we impart to our students.

We hope this issue brings to you a wealth of learning and diverse experiences from our country and abroad. Happy reading!

\section{Declarations}

Conflict of interest The authors declare that they have no conflict of interest.

Ethical standard statement This article does not contain any studies with human or animal subjects performed by the any of the authors.

Informed consent For this type of study informed consent is not required.

\section{References}

1. Vaidya, S. V., Jogani, A. D., Pachore, J. A., et al. (2021). India joining the world of hip and knee registries: present status-a leap forward. Indian Journal of Orthopaedics, 55, 46-55.

2. Nisar, S., Palan, J., Rivière, C., Emerton, M., \& Pandit, H. (2020). Kinematic alignment in total knee arthroplasty. Efort Open Reviews, 5, 380-390. 
3. Jones, C. W., \& Jerabek, S. A. (2018). Current role of computer navigation in TKA. Journal of Arthroplasty, 33(7), 1989-1993.

4. St Mart, J. P., \& Goh, E. L. (2021). The current state of robotics in total knee arthroplasty. EFORT Open Reviews, 6(4), 270-279. https://doi.org/10.1302/2058-5241.6.200052

5. Mohanty, S. S., Mohan, H., Rathod, T. N., \& Kamble, P. (2020). Patient satisfaction related outcome after total hip arthroplasty; does bearing surface play a role? Journal Clinical Orthopaedics and Trauma, 11(Suppl 2), S196-S200.

6. Morton, J., Eftekhary, N., Schwarzkopf, R., \& Vigdorchik, J. M. (2018). The spinopelvic relationship made simple: what every hip surgeon needs to know to prevent instability in high-risk patients undergoing total hip arthroplasty. Seminars in Arthroplasty, 29, 274-281.
7. Wasterlain, A. S., Karan Goswami, S., Ghasemi, A., \& Parvizi, J. (2020). Current concepts review, diagnosis of periprosthetic infection, recent developments. Journal of Bone and Joint Surgery American Volume, 102, 1366-1375. https://doi.org/10.2106/JBJS. 19.00598

Publisher's Note Springer Nature remains neutral with regard to jurisdictional claims in published maps and institutional affiliations. 